\title{
Coupled X-ray fluorescence and X-ray absorption spectroscopy for microscale imaging and identification of sulfur species within tissues and skeletons of scleractinian corals
}

\author{
Gabriela A. Farfan ${ }^{\dagger,}$, Amy Apprill ${ }^{\ddagger}$, Samuel M. Webb ${ }^{\S}$, Colleen M. Hansel ${ }^{\ddagger *}$
}

${ }^{\dagger}$ MIT-WHOI Joint Program in Oceanography/Applied Ocean Science and Engineering, Woods Hole Oceanographic Institution, Woods Hole, MA 02543, United States

${ }^{ \pm}$Department of Marine Chemistry and Geochemistry, Woods Hole Oceanographic Institution, Woods Hole, MA 02543, United States

${ }^{\S}$ Stanford Synchrotron Radiation Lightsource, SLAC National Accelerator Laboratory, Menlo Park, CA 94025, United States

\begin{abstract}
Identifying and mapping the wide range of sulfur species within complex matrices presents a challenge for understanding the distribution of these important biomolecules within environmental and biological systems. Here, we present a coupled micro X-ray fluorescence ( $\mu \mathrm{XRF}$ ) and X-ray absorption near edge structure (XANES) spectroscopy method for determining the presence of specific sulfur species in coral tissues and skeletons at high spatial resolution. By using multiple energy stacks and principal component analysis of a large spectral database, we were able to more accurately identify sulfur species components and distinguish different species and distributions of sulfur formerly unresolved by previous studies. Specifically, coral tissues were dominated by more reduced sulfur species, such as glutathione disulfide, cysteine and sulfoxide, as well as organic sulfate as represented by chondroitin sulfate. Sulfoxide distributions were visually correlated with the presence of zooxanthellae endosymbionts. Coral skeletons were composed primarily of carbonate-associated sulfate (CAS), along with minor contributions from organic sulfate and a separate inorganic sulfate likely in the form of adsorbed sulfate. This coupled XRF-XANES approach allows for a more accurate and informative view of sulfur within biological systems in situ, and holds great promise for pairing with other techniques to allow for a more encompassing understanding of elemental distributions within the environment.
\end{abstract}

Scleractinian corals compose the structural and biological framework of corals reefs, which are vital ocean ecosystems that host hotspots of biodiversity, provide ample marine resources and help to drive tourism-based economies. ${ }^{1}$ Sulfur (S) is ubiquitous as an essential element for life, as it is involved in organosulfur compounds such as amino acids (cysteine, methionine) and as a cofactor in proteins such as glutathione disulfide. It is also common in many oxidation states as building blocks for minerals ranging from elemental sulfur, to reduced sulfide minerals (pyrite) and oxidized sulfate minerals (gypsum). In corals, sulfur is present in both the living tissues and non-living skeletal components as both organic and inorganic phases. Thus, sulfur in corals has been studied from many perspectives, including characterizing sulfate incorporations into aragonitic skeletons as a way to better understand skeletal growth and deduce the role of organics in biomineralization mechanisms, ${ }^{2,3,4,5,6}$ quantifying dimethylsulphoniopropionate (DMSP) production in the tissues and endosymbionts as an elevated temperature stress signal during coral bleaching events, ${ }^{7}$ and measuring $\mathrm{S}$ isotopes in carbonate-associated sulfate (CAS) as a paleoproxy. ${ }^{8}$ Furthermore, sulfur is an important trace metal ligand and its cycling and attenuation in coral reefs may be intricately linked to bio-limiting trace metal cycles that control biological productivity in these oligotrophic ecosystems. Understanding the spatial distributions of the many sulfur species in corals is thus essential to further unlocking questions surrounding these biogeochemical processes.

The utility of synchrotron radiation techniques for speciating sulfur and investigating the role of sulfur molecules and ligands in situ has proven useful in environmental contexts such as organic matter, ${ }^{9}$ metabolites,${ }^{10}$ soils, ${ }^{11}$ brachiopods, ${ }^{12}$ mollusks, ${ }^{13}$ and coral skeletons. ${ }^{3,6,14}$ Specifically, synchrotronbased studies on corals (mostly on precious red octocorals) have identified a range of sulfur species present in corals via single-point X-ray absorption near edge structure (XANES) spectroscopy. While this approach tells us what $S$ functional groups are most likely present in corals as a whole, the extent to which each species plays a role in the coral and where it is distributed depends on visual correlations. ${ }^{4,6,14}$ Some studies have taken this approach further and mapped the distributions of reduced versus oxidized sulfur regions by using micro $\mathrm{X}$ ray fluorescence spectroscopy ( $\mu \mathrm{XRF}$ ) raster maps to overlay two single-energy maps representing reduced versus oxidized $\mathrm{S}$ energies. These studies observed that oxidized $\mathrm{S}$ regions visually correlated with skeletal regions and are made up of sulfate-dominated XANES points, while reduced sulfur regions align with the coenenchyme and comprise XANES points of reduced organosulfur species. ${ }^{6,14}$

In this study, we extend the capacity for pairing synchrotron-based XANES with $\mu$ XRF maps to better interrogate the distribution of individual sulfur functional groups within skeletons and specific coral tissues at micron-scale resolution. Similar approaches have been successful in evaluating the distributions and bonding environments of metals within other environmental and geological systems. ${ }^{15,16}$ For instance, Mayhew et al., ${ }^{17}$ introduced a method combining Fe XANES with Fe multiple energy $\mu$ XRF maps to determine oxidation state, speciation, and distributions of Fe in heterogeneous samples of fluid-mineral interactions that resulted in the successful mapping of a wide range of reduced and oxidized Fe species within a single sample. Our study builds on previous sulfur in coral synchrotron-based studies that map reduced sulfur (or organic sulfur) versus oxidized sulfur (or sulfate) ${ }^{6,14}$ by adapting the multiple energy (ME) $\mu$ XRF mapping technique by Mayhew et al. ${ }^{17}$ with extensive XANES points to obtain high-resolution spatially-resolved maps of specific sulfur species in tissues and skeletons. Using these $\mathrm{S}$ species-specific maps, we detail how sulfur species relate to specific coral tissues and endosymbiont distributions in aragonitic scleractinian corals. 


\section{MATERIALS AND METHODS}

Samples. We obtained scleractinian coral samples of Diploria labyrinthiformis from Carmabi, Curaçao, Porites astreoides from Water Factory, Curaçao, and Diploastrea heliopora from Yap Island, Federated States of Micronesia. Samples were rinsed of excess mucus, preserved in trace-metal grade paraformaldehyde (Electron Microscopy Sciences), diluted with sterile seawater ( $4 \%$ final concentration), and stored at $4{ }^{\circ} \mathrm{C}$. Samples were later partially dehydrated and kept in $70 \%$ ethanol at $-20^{\circ} \mathrm{C}$. Samples from Carmabi, Curaçao represent corals growing in more anthropogenically-influenced waters (some samples from Carmabi were growing on metal structures) while those from Water Factory, Curaçao, Florida Keys, USA and Yap Island, Micronesia represent corals grown in a range of other geographic locations.

Thin Sections. Tissues and skeletons were impregnated with a low-sulfur and low-fluorescence Struers ${ }^{\circledR}$ EpoFix epoxy to preserve the tissue morphologies for thin-sectioning. The samples were cut vertically across the polyps (to expose tissue layers and cross-sections of coral septa) and polished into $30 \mu \mathrm{m}$-thick standard geologic thin-sections using Beta Diamond Inc. products and a polishing paper with minimal trace metals (confirmed via $\mu \mathrm{XRF}$ ). All thin-sections were made by High Mesa Petrographics, Los Alamos, New Mexico. Optical images (Life Technologies, EVOS FL Auto, Woods Hole Oceanographic Institution) and environmental scanning electron microscopy (ESEM) (Hitachi TM300, Woods Hole Oceanographic Institution) images were taken of the thin-sections to identify regions of interest for $\mu \mathrm{XRF}$ mapping and to confirm that these regions contained minimal algal boring or other alterations.
Low Energy X-ray $\boldsymbol{\mu X R F}$ mapping. For determining in situ sulfur distributions, Synchrotron-based fluorescence maps $(\mu \mathrm{XRF})$ and sulfur X-ray absorption near edge spectroscopy ( $\mathrm{S}$ XANES) were measured at BL 14-3 at the Stanford Synchrotron Radiation Lightsource (SSRL) using a $\operatorname{Si}(111)(\Phi=90)$ double crystal monochromator. The monochromator was calibrated at the thiol pre-edge energy of a sodium thiosulfate powder to be $2472.02 \mathrm{eV}$. The fluorescence lines of interest were measured with a Si Vortex Si drift detector (SII Nano Technology) using Xspress3 pulse processing electronics (Quantum Detectors) that deliver superior count rates without significant dead times. The X-ray beam was focused using Kirkpatrick-Baez (K-B) mirrors to a size of 5 x $5 \mu \mathrm{m}$ at a flux of $\sim 2 \times 10^{10}$ photons per second. To determine sulfur speciation at specific points within the sample, absorption spectra ( $S$ XANES) were collected from 2460 to $2536 \mathrm{eV}$ at $0.2 \mathrm{eV}$ steps to capture the range of energies for the absorption edges of possible common sulfur compounds. Multiple sweeps were acquired at each point to confirm that the absorption edge energies did not shift to lower energies due to photoreduction by beam damage to the sample.

To map the distributions of different sulfur species within the corals, fluorescence maps were obtained at multiple energies $(2472.8,2473.9,2475.1,2476.4,2481.4,2482.4$, and $2484.7 \mathrm{eV}$, as well as 2488.0 for normalization). These energies were chosen based on unique absorption edge positions of common biologically-relevant sulfur species (Figure 1) and preliminary S XANES points taken across the $P$. astreoides sample from Water Factory, Curaçao. Multiple energy (ME) $\mu \mathrm{XRF}$ maps were collected with a 5 x $5 \mu \mathrm{m}$ pixel resolution and a dwell time of $50 \mathrm{~ms}$ per pixel.

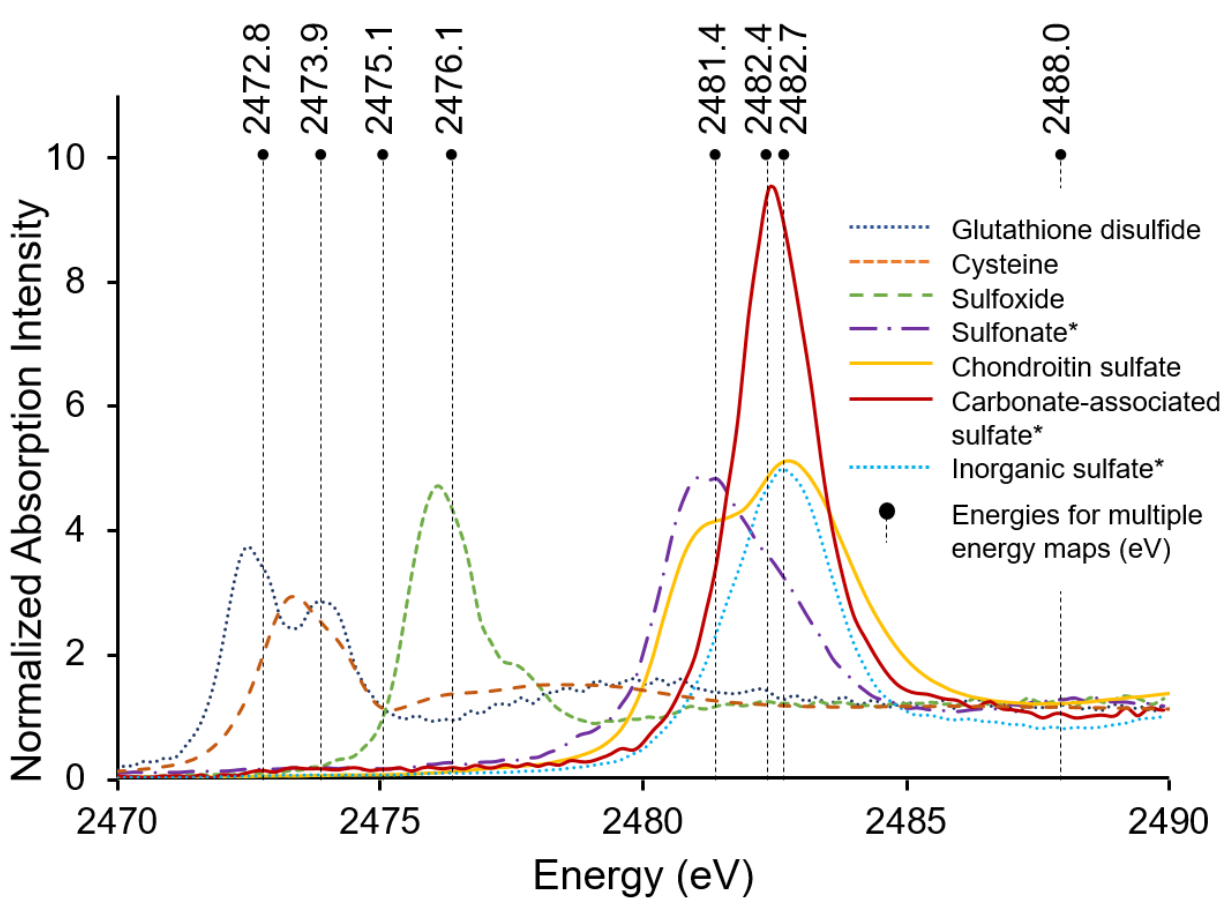

Figure 1. Normalized standard S XANES spectra selected for fitting multiple energy (ME) sulfur maps (with the exception of inorganic sulfate). Energies (eV) selected for ME maps are marked with filled black circles and dashed lines through the spectra. Asterisk denotes $\mathrm{S}$ XANES spectra used as internal standards obtained directly from our coral samples and identified as an end-members via PCA analysis. 
Fitting Sulfur ME Maps with S XANES. In order to successfully describe the distribution of sulfur bonding environments in a heterogeneous coral sample using ME maps, it was crucial to select the appropriate number and variety of standard sulfur compounds that best describes the coral sulfur system. Rather than only relying on the main absorption peak energies of a set of standards and assuming that XRF maps collected at those energies reflect the distributions of those standards, ${ }^{4,6}$ we utilized a stack of ME maps and fit standard S XANES spectra to the entire stack of maps to provide a more accurate distribution of each sulfur standard. Utilizing a stack of energies and several representative absorption features, rather than only one dominant peak, eliminates bias from overlapping XRF signals from neighboring lower energies and allows for better resolution between standards that have main peaks at similar energies. This method of using XANES spectral analysis to fit XANES to ME maps was first reported by Mayhew et al., ${ }^{17}$ to investigate the distribution of iron compounds in fluid-mineral reactions and is also similarly employed in scanning X-ray transmission microscopy near edge X-ray absorption fine structure (STXM-NEXAFS) spectroscopic imaging. ${ }^{18,19}$

Properly fitting ME maps with XANES, as described by Mayhew et al., 2011 and as modified in this study for sulfur, requires combining PCA analysis of ME $\mu$ XRF maps, PCA of XANES, and linear combination fitting of XANES spectra using XANES standards and XANES PCA end-members using SIX-PACK ${ }^{20}$ and Microanalysis Toolkit ${ }^{21}$ software in order to identify the number of components required to properly fit the stack of maps with the most representative standards for the sample with the following steps: 1) ME S $\mu$ XRF maps were collected at energies selected based on positions on preliminary S XANES spectra that differed significantly from one another. These energies correspond to main peak positions of $S$ XANES standards (Figure 1) used to fit the ME maps in the following steps. These multiple energy maps were stacked using Microanalysis Toolkit ${ }^{21}$. 2) 30-43 S XANES were collected per sample to represent the variability found in the samples, as guided by distinct components identified via PCA of the stacked ME maps, Kmeans clustering, and visual features identified in optical microscopic analysis of the thin-sections (Supporting Information (SI), Figures S1, S2). S XANES spectra of typical coral tissues and skeletons are presented in Figure 2 with linear combination fits using the $\mathrm{S}$ species used to represent the system in Figure 1. 3) PCA analyses of the S
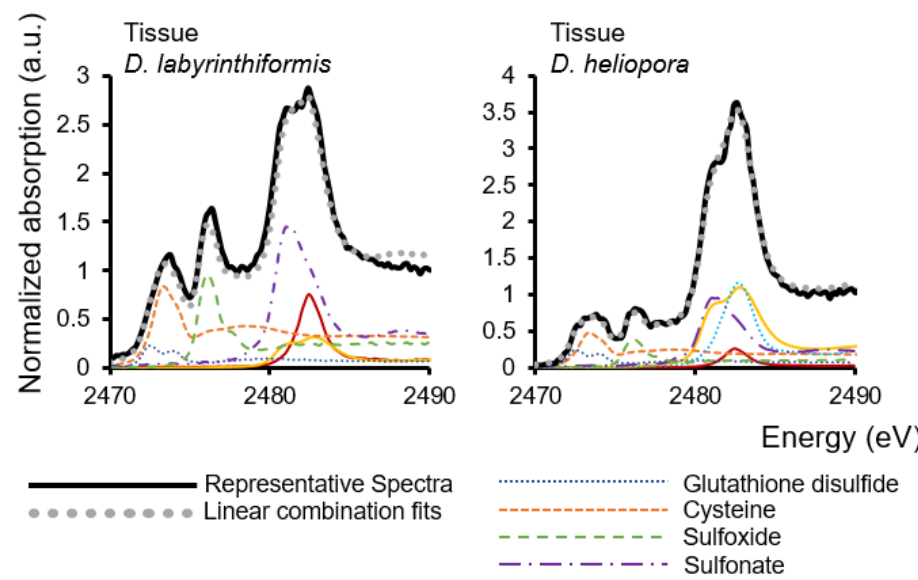

Figure 2. Linear combination fits (grey dotted lines) of micro-S XANES spectra from typical tissues and skeletons of D. labyrinthiformis and $D$. heliopora (black lines) underlain by the relative contributions from S XANES standards used for the fitting.

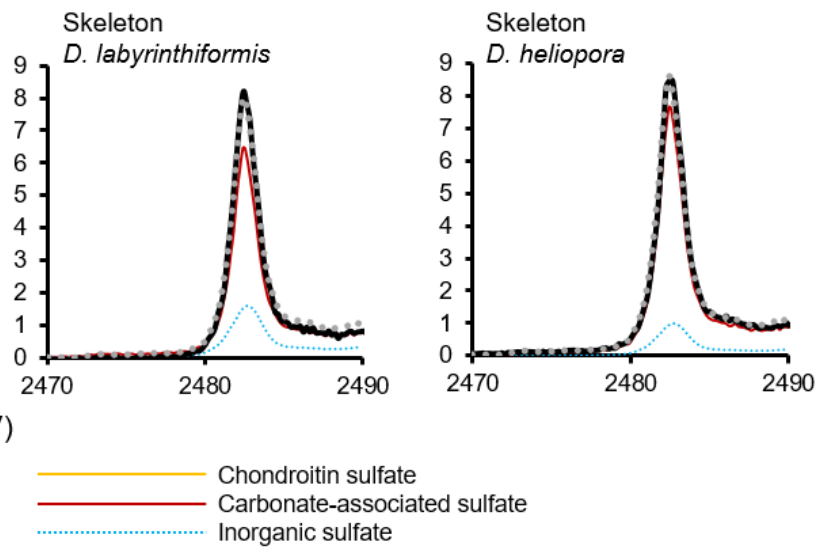

XANES taken from the ME sulfur $\mu$ XRF maps were conducted (at a 2470 to $2490 \mathrm{eV}$ span) using the SIXPACK software package ${ }^{20}$. The number of principal components ( 6 to 7 in this study) was identified from the S XANES by determining where additional components no longer contributed to the signal and the scree plot reached a plateau. 4) Next, end-member spectra identified by PCA component score plots were fit using linear combination fitting in SIXPACK ${ }^{20}$ with $\mathrm{S}$ XANES standards from sulfur spectral libraries acquired from the European Synchrotron Radiation Facility (it includes more than 60 sulfur standards, including a broad spectrum of inorganic and organic sulfur species) and a set of spectra from our personal S XANES library measured at SSRL, BL14-3, including glutathione, cysteine and sulfoxide. Spectra obtained from the ESRF database are consistent with those collected at SSRL in peak shape and position (SI, Figure S3). Overall, most end-members were describable using these standards, however a few end-members were not sufficiently described by the standard library compounds and were thus selected to be internal standards for fitting the coral ME maps. These spectra include a sulfonate-like compound, carbonate-associated sulfate, and another inorganic sulfate, all three of which are presented with an asterisk in Figure 1. In total we selected seven (the number determined by Step \#3) S standard compounds representing different $S$ bonding environments needed to describe the system. S XANES of these seven compounds are plotted in Figure 1.5) Target transforms of these standard $S$ XANES spectra to the seven principal components identified via PCA in part 3 (using PC Analysis in SIXPACK ${ }^{20}$ ) were used to confirm the effectiveness of the seven standard $S$ XANES spectra in defining the coral sulfur system. 6) Due to some maps being over-constrained with seven standards, six of the seven standard S XANES (Figure 1) were fit to the stack of ME maps using MicroAnalysis Toolkit ${ }^{21}$, resulting in maps of the distributions of six standard sulfur species (Figure 3). It is important to stress that while PCA analysis, linear combination fittings, and target transforms are used to select the final standard S XANES (steps 2, 4, 5, and 6), these S XANES are real data from discrete points on our samples or from data repositories and they are not subject to any statistical treatments or changes that would alter their peak position or shape. 7) In order to validate the accuracy of our fitted maps, we plotted the contributions of S standards to all of our $\mathrm{S}$ XANES collected in a sample by linear combination fitting, 
versus the contributions of the standards determined by the fitted maps for each position where the S XANES points were collected (SI, Figures S1, S2, S4, Table S1). A highly accurate fit should be a 1:1 line between linear combination fits versus map fits.

\section{RESULTS}

Using our approach for fitting ME $\mu$ XRF maps with real S XANES spectra, we mapped the distributions of six $\mathrm{S}$ bonding environments across heterogeneous coral samples using $S$ XANES of the following standard compounds: glutathione disulfide (hereinafter referred to as glutathione), cysteine, sulfoxide, a sulfonate-like S XANES spectrum (hereinafter referred to as sulfonate), organic sulfate (represented by a chondroitin sulfate standard, hereinafter referred to as organic sulfate), CAS, and another form of sulfate that is unlike CAS or chondroitin sulfate (hereinafter referred to as inorganic sulfate) (Figure 1). Four of these standards were selected from spectral libraries, while sulfonate, CAS and inorganic sulfate components are internal standards identified from coral $\mathrm{S}$ XANES collected in this study (marked with an asterisk in Figure 1). Upon validating our results, we removed the inorganic sulfate standard from our map fits because the sevenstandard fits proved to be over-constrained, however the inorganic sulfate standard was kept in the S XANES linear combination fits.

Reduced sulfur species. Three reduced sulfur species were identified via PCA analysis and spectral fitting - glutathione, cysteine and sulfoxide - all of which have biological relevance in coral tissues. Of the various glutathione standards, the oxidized form, glutathione disulfide, fit the data best and was used to represent glutathione-like molecules. The amino acids, cysteine and methionine, have overlapping absorption edges, thus only one was used for the fitting. Cysteine was chosen as the representative species for these absorption energies because it fit better in the target transform analyses. A sulfoxide standard was selected as representative for dimethylsulfoniopropionate (DMSP). Finally, a distinct sulfonate-like endmember was also identified from the coral S XANES spectra PCA analysis (2481.20 eV, labeled as "sulfonate"). Sulfonate was identified as a minor component in some maps and thus the spectrum was included in the fit, however it is important to note that these signals came from areas that may represent contamination from either detritus or a filled bubble in the epoxy and polishing process (SI, Figure S5).

Sulfate species. Our study identified three different bonding environments for sulfate in coral skeletons and tissues: CAS (absorption edge peak at $2482.44 \mathrm{eV}$ ), another inorganic sulfate $(2482.65 \mathrm{eV})$ and organic sulfate (strongest peak at $2482.74 \mathrm{eV}$ split with a minor peak at $2481.4 \mathrm{eV}$, represented by chondroitin sulfate). We designate our dominant sulfate signal associated with the aragonite skeletons as CAS because its absorption peak is shifted to lower energies than the gypsum standard and the other inorganic sulfate, as would be expected for a structurally (substituted) bound sulfate within the aragonite lattice. The inorganic sulfate end member is also dissimilar to gypsum because its S XANES spectrum lacks a post edge feature observed in gypsum at $2486 \mathrm{eV}$. While the bonding environment of this other inorganic sulfate species is un- known at present, it is likely a sorbed species within the skeleton based on the higher energy shift. Despite gypsum being used as a representative sulfate standard in previous studies, ${ }^{4,6}$ skeletal sulfate spectra fit more accurately with our CAS and inorganic sulfate end-members, rather than gypsum, using linear combination fitting in SIXPACK (Figure 2). Furthermore, gypsum was not included in our fits because it was not observable in coral skeletons via Raman spectroscopy ${ }^{3}$ and $S$

XANES studies of sulfate in carbonates confirm that sulfate is not incorporated as gypsum or anhydrite in carbonates. ${ }^{21}$

Fitted ME $\boldsymbol{\mu}$ XRF Maps. Fitted ME $\mu$ XRF maps of sulfur species distributions are presented as heat maps (Figure 3 ) and tricolor plots (Figure 4). The major sulfur components within the tissue regions are sulfur species most similar to glutathione disulfide, cysteine, sulfoxide and organic sulfate. Cysteineand glutathione-like compounds are mostly anti-correlated throughout the tissues with glutathione occurring in concentrated points, especially in $P$. astreoides (Figure 4B). Sulfoxide-like species are loosely correlated with cysteine and glutathione distributions and anti-correlated with organic sulfate. Zoomed-in views of tissue regions rich in zooxanthellae in the gastroderm (labeled g), correlate visually with distributions of sulfoxide (Figure 5). CAS is dominant in the skeletons and mostly anti-correlated with all other sulfur species.

\section{DISCUSSION}

Comparison to previous XRF mapping techniques. Previous studies have provided spatial maps detailing the distribution of oxidized and reduced S regions of corals. ${ }^{6,14}$ Here, we expand on synchrotron-based paired XANES- $\mu$ XRF methods to identify and map specific $S$ functional groups and species in situ at high spatial resolution. Further, rather than relying on single-energy maps that contain background fluorescence signals from lower energy absorption peaks or subtracting reduced energy maps from total energy maps, ME fitting separates the signals from individual species and allows for more accurate species-specific mapping. To aid in presenting this distinction, comparisons of single energy maps versus ME fitted maps are included in the Supporting Information (Figure S6) along with validation steps for our fitting method (Figures S1, S2, S4; Table S1). PCA analysis of an extensive XANES library further increases the accuracy of this method by properly constraining the number of components necessary to describe the system. Lastly, smaller energy step-sizes $(0.2 \mathrm{eV})$ in XANES spectra and higher-resolution $\mu$ XRF maps $(5 \times 5$ $\mu \mathrm{m}$ steps) allowed for differentiating between previously unresolved sulfate variations in corals. In combination, this approach allowed for high-resolution, species-specific maps of sulfur within and across the tissue-skeleton interface in corals. In our validation step, compound compositions extracted from our map fits compared to the XANES linear combination fits of our standards in our D. heliopora example did not always fit ideal 1:1 lines (SI, Figure S4). This is likely due to the highly heterogeneous nature of the sample and the relatively low concentrations of certain compounds throughout the $D$. heliopora example that makes fitting more difficult.

Sulfur species in tissues. Four representative sulfur compounds, glutathione, cysteine, sulfoxide and organic sulfate, represent the array of sulfur species observed in the coral 


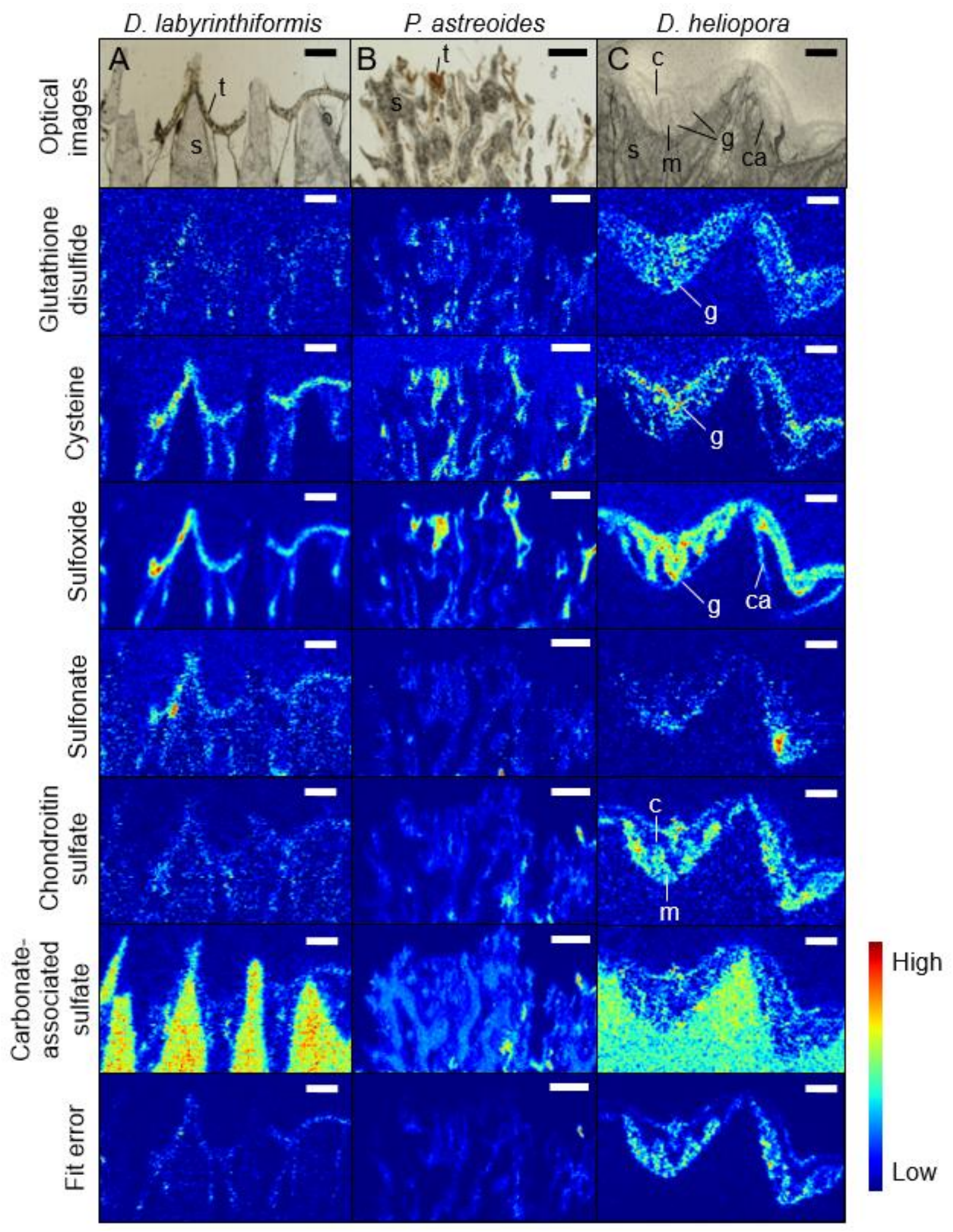

Figure 3. Multiple energy (ME) sulfur $\mu$ XRF maps fit with the sulfur XANES standards (Fig. 1) presented as heat maps (blue= low relative concentration, red= high relative concentrations) of A. D. labyrinthiformis, Carmabi, Curaçao (scale bar $=100 \mu \mathrm{m}$ ), B. P. astreoides, Water Factory, Curaçao (scale bar $=200 \mu \mathrm{m}$ ) and C. D. heliopora, Yap Island, Federated States of Micronesia $(\mathrm{scale}$ bar $=100 \mu \mathrm{m})$. Lessabundant skeletal chondroitin sulfate and inorganic sulfate features in the skeletons are pointed out with yellow arrows. Top optical images illustrate the location of the skeleton $(\mathrm{s}=$ skeleton $)$ and tissues $(\mathrm{t}=$ tissue $)$. Specific tissue layers are also labeled on $\mathrm{C}$ : $\mathrm{g}=\mathrm{gastroderm}, \mathrm{m}=$ mesoglea, $\mathrm{c}=$ coelenteron, $\mathrm{ca}=$ calicoderm. 


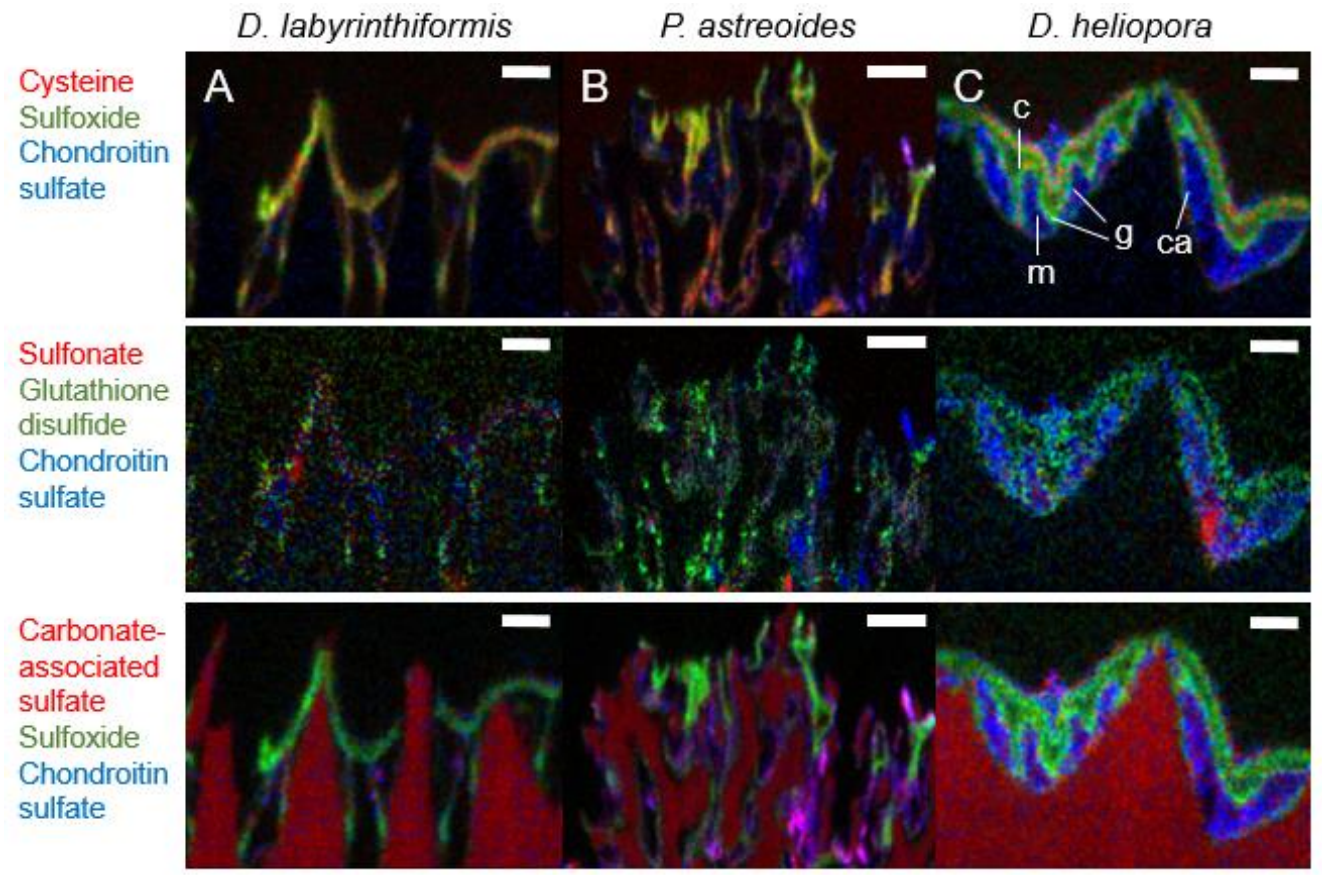

Figure 4. Tricolor plots of distributions of different sulfur species in A. D. labyrinthiformis, Carmabi, Curaçao (scale bar $=100 \mu \mathrm{m})$, B. $P$. astreoides, Water Factory, Curaçao (scale bar $=200 \mu \mathrm{m}$ ) and C. D. heliopora, Yap Island, Federated States of Micronesia (scale bar $=100$ $\mu \mathrm{m})$. The location of the skeleton is indicated by the bottom panel as carbonate-associated sulfate. Specific tissue layers are labeled on C: $\mathrm{g}$ $=$ gastroderm, $\mathrm{m}=$ mesoglea, $\mathrm{c}=$ coelenteron, $\mathrm{ca}=$ calicoderm .

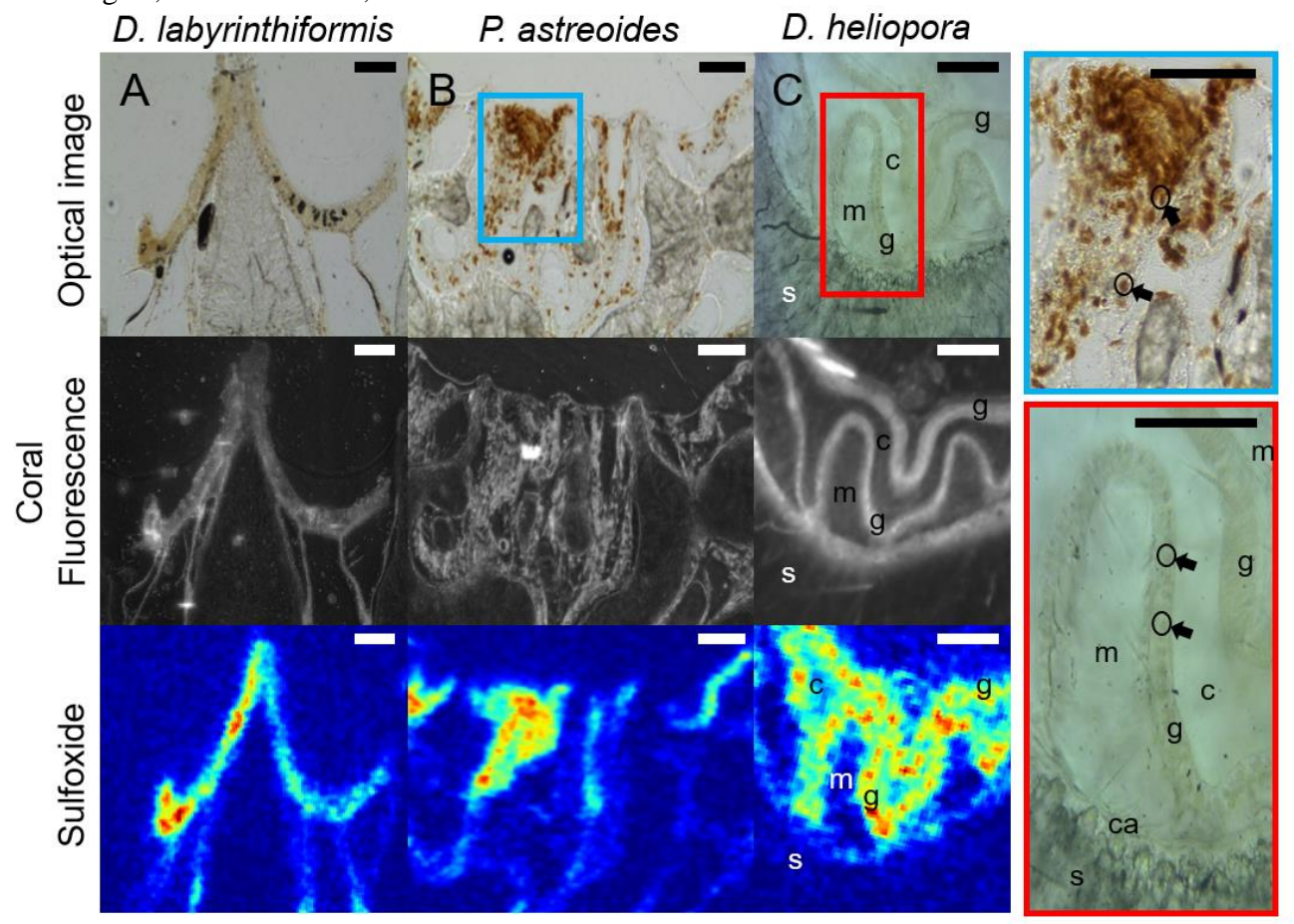

Figure 5. Correlations observed between the distributions of zooxanthellae and tissues (optical and fluorescence maps) with sulfoxide ( $\mu$ XRF maps). Samples include A). D. labyrinthiformis, Carmabi, Curaçao, B). P. astreoides, Water Factory, Curaçao, and C). D. heliopora, Yap Island, Federated States of Micronesia. Black open circles with arrows point out zooxanthellae. Specific tissue layers $(\mathrm{g}=$ gastroderm, $\mathrm{m}=$ mesoglea, $\mathrm{c}=$ coelenteron, $\mathrm{ca}=$ calicoderm) and skeleton $(\mathrm{s})$ and are labeled on $\mathrm{C}$. Scale bars are $100 \mu \mathrm{m}$.

tissues explored here (Figures 1 \& 3). Until recently, synchrotron-based studies had only utilized cysteine and methionine as standards to describe the reduced sulfur species observed in the coenenchyme of octocorals; ${ }^{6,23}$ however, a recent study has identified or inferred a similar assemblage of sulfur species in octocoral tissues (i.e. glutathione, thioether, disulfide, sulfoxide, sulfonate and sulfate) as we observe in scleractinian corals, and which we use to fit our ME maps. ${ }^{14}$ Our fitted ME maps and S XANES spectra demonstrate that overall abundances and distributions of these selected sulfur bonding environments remain similar across coral species (Figures $2 \& 3$ ). 
The distributions of these different $\mathrm{S}$ species point to their biological roles within coral tissues. Individual tissue layers were identified by referencing Allemand et al. ${ }^{24,25}$ The most distinct sulfur feature identified across the coral tissues measured in this study is the presence of a sulfoxide-like compound concentrated in the gastroderm, the layer of tissue which hosts zooxanthellae, and which co-occurs with high concentrations of zooxanthellae (Figure 5, gastroderm labeled with a "g"). This sulfoxide signal is likely dimethylsulfoniopropionate (DMSP), an antioxidant which is produced at elevated levels by zooxanthellae, as well as by the coral tissues themselves (but to a lesser degree) when these organisms are exposed to oxidative and heat stress. ${ }^{7,26}$ This sulfoxide is also correlated with a cysteine-like component, a sulfur-bearing amino acid, which occurs in high concentrations in protein-rich regions, such as around zooxanthellae or other metabolically-active coral tissues (Figures $4 \& 5$ ). One difference is that that sulfoxide is mostly restricted to the gastroderm while the cysteine-like species is also present in other areas of the tissue (Figures 3-5). Cysteine and a sulfur species resembling glutathione both occur throughout the tissues, however, they are loosely anti-correlated. A glutathione-like species is likewise present in the gastroderm but also occurs as hot spots in other tissues likely associated with high concentrations of endosymbiotic bacteria known to use glutathione for their sulfur metabolisms and redox regulation, especially in $P$. astreoides (Figures 3B $\& 4 B) .{ }^{27}$ Organic sulfate (represented by a chondroitin sulfate $\mathrm{S}$ XANES standard) is anti-correlated with all of the reduced sulfur species, likely because chondroitin sulfate is a common structure-building polysaccharide in animal tissues and in our samples is present in the mesoglea, an extracellular matrix composed predominantly of water and collagen most clearly detailed in D. heliopora (Figures 3C \& 4C). ${ }^{24}$ Furthermore, this organic sulfate is not associated with the unicellular endosymbionts correlating with the sulfoxide, cysteine and glutathione distributions.

Sulfur in the skeletons. This study introduces a method to speciate and localize the variety of sulfate bonding environments in the skeleton. We observe that carbonate-associated sulfate (CAS) is the dominant sulfur signal observed in the aragonitic coral skeletons and that it is especially concentrated along centers of calcification (Figure 3 ). This finding corroborates previous studies that focused on mapping sulfur in coral skeletal microstructures and similarly observed a higher concentration of sulfate in centers of calcification. ${ }^{3}$ Our CAS is distinguishable via $\mathrm{S}$ XANES from another inorganic sulfate species within the skeleton and from gypsum. The presence of CAS corroborates previous studies of other aragonite and calcite coral skeletons suggesting that skeletal sulfur is composed predominantly of substitutions of $\mathrm{SO}_{4}{ }^{2-}$ for $\mathrm{CO}_{3}{ }^{2-}$ in the crystal structure, ${ }^{4,6,22}$ despite the incorporation of $\mathrm{SO}_{4}{ }^{2-}$ into the aragonite structure being energetically unfavorable compared to other carbonates such as calcite. ${ }^{28,29,30}$ This inorganic substitution of sulfate for carbonate groups in the crystal structure was originally proposed as a mechanism for the incorporation of sulfate in marine biogenic carbonates based on IR measurements and is still considered to be the most likely method for sulfate incorporation in biogenic carbonates. ${ }^{31}$ Since then, postulated mechanisms for sulfur incorporation in carbonates have included sulfide, sulfite and sulfate substitutions for carbonate, sulfate-bearing mineral inclusions, native sulfur incorporations, sulfate fluid inclusions, adsorption of sulfate onto carbonate surfaces and intra- and inter-crystalline S-bearing organic matter. ${ }^{14,22,28} \mathrm{CAS}$ is also described in calcite and calcitic corals as structurally substituted sulfur (SSS). ${ }^{5,32}$

Another inorganic sulfate component was clearly differentiated from the CAS by a $0.2 \mathrm{eV}$ shift in the absorption edge for these two species (Figure 1), however it was present in too low of concentrations to be included in our map fits (Figure 3 ). We propose that this is likely a sorbed sulfate species within the skeleton. By using PCA on the extensive collection of $S$ XANES points to identify representative end-member species in the corals, this minor sulfate species was able to observed and identified.

Results of our fitted ME maps reveal a minor presence of organic sulfur species in the skeletons which is anti-correlated with CAS. This observation is consistent with previous studies that identified both inorganic sulfate and organic sulfur species in calcite and aragonite coral skeletons (Figure 3). ${ }^{4,5,6,22}$ As these are natural, heterogeneous samples, this organic sulfate signal in the skeletons could be associated with boring algae or with a skeletal organic matrix. While organic sulfate has been proposed as both a major ${ }^{3}$ and minor ${ }^{4,6}$ sulfur component within coral skeletons, our S XANES observations, along with more recent synchrotron-based and complementary studies, suggest that organic sulfate is a minor sulfur component in coral skeletons and that CAS comprises most of the observed sulfur signal. Mass balance calculations from previous studies indicate that structurally-substituted sulfur and organo-sulfur species were present at approximately a 20:1 ratio in the calcite skeletons of $P$. japonicum ${ }^{4,5,33}$ with an upper bound for total sulfate in biogenic carbonates as $5000 \mathrm{ppm}$ in aragonitic coral skeletons $^{22}$ and $3100 \pm 400 \mu \mathrm{g} / \mathrm{g}$ in calcitic Corallium rubrum skeletons. ${ }^{5}$ Despite the lack of a major organic sulfate presence in the skeletons, our thin-sectioned samples of combined skeleton and tissues reveal that organic sulfate species are much more pronounced in coral tissues relative to in the skeleton (Figures $3 \& 4$ ).

\section{CONCLUSIONS}

Synchrotron-based methods offer a unique approach to observing in situ elemental distributions and bonding environments in complex environmental samples. Here, sulfur $\mu$ XRF ME maps with end-members and standards from extensive $S$ XANES observations and analyses allowed for distinguishing between different species and distributions of sulfur formerly unresolved by previous studies. Specifically, tissues are composed of reduced sulfur species similar to glutathione, cysteine and sulfoxide, as well as organic sulfate, while skeletal sulfur is dominated by CAS with very minor amounts of (ad)sorbed sulfate and organic sulfate. Sulfur distributions follow consistent spatial patterns in coral tissues and skeletons, regardless of species and locality. For example, CAS is concentrated along centers of calcification, along the septa of skeletons, while sulfoxide is located in tissues that are especially concentrated with zooxanthellae. Our method of coupling $\mu$ XRF with XANES produces more accurate representations of sulfur species distributions compared to single-energy maps or XANES fittings with $\mu \mathrm{XRF}$ maps that use less-representative standards, such as gypsum to represent sulfate, from spectral databases. Future directions for this method could include combining these low-energy X-ray maps with metal species data obtained on high-energy beamlines as well as microbial distributions using spatial biological techniques such as fluorescent in situ hybridization probes. 


\section{ASSOCIATED CONTENT}

\section{Supporting Information}

The Supporting Information is available free of charge on the ACS Publications website.

Figures of example S XANES spectra, linear combination fits, validation plots, multiple energy maps versus maps with $S$ XANES fits, example of sulfonate contamination, and a table of standard compound contributions (PDF)

\section{AUTHOR INFORMATION}

\section{Corresponding Author}

* Email: chansel@whoi.edu, Phone: 508.289.3738

ORCID: 0000-0002-3506-7710

\section{Author Contributions}

G.A.F. collected and analyzed all $\mu$ XRF and XANES data. S.W. provided expertise on the ME map fitting method and helped interpret final maps with C.M.H and G.A.F. A.A. provided the coral samples and interpreted relevance to biological tissues with C.M.H. and G.A.F. G.A.F wrote the manuscript with assistance from C.M.H. All authors have given approval to the final version of the manuscript.

\section{ACKNOWLEDGMENTS}

We thank Ray Dalio for funding the Micronesian expedition and K. Hughen, M. Neave, J. Ossolinski, A. Santoro, C. Smith, and the crew of the M/V Alucia for sampling support and the Federated States of Micronesia for collection permits FM12-11-03S and FM12-11-05S. In Curaçao, we are grateful to Mark Vermeij, Kristen Marhaver and the CARMABI Research Institute for logistical support and permit AN-001 issued by Curaçao.

Use of the Stanford Synchrotron Radiation Lightsource, SLAC National Accelerator Laboratory, is supported by the U.S. Department of Energy, Office of Science, Office of Basic Energy Sciences under Contract No. DE-AC02-76SF00515. The SSRL Structural Molecular Biology Program is supported by the DOE Office of Biological and Environmental Research, and by the National Institutes of Health, National Institute of General Medical Sciences (including P41GM103393). The contents of this publication are solely the responsibility of the authors and do not necessarily represent the official views of NIGMS or NIH.

The authors would also like to acknowledge Courtney Krest (SSRL) for help with BL 14-3, Horst Marschall and Adam Sarafian (WHOI) for help with SEM measurements and Anne Cohen (WHOI) and Konrad Hughen (WHOI) for helpful coral discussions. This material is based upon work supported by the National Science Foundation Graduate Research Fellowship under Grant No. 1122374 and a Ford Foundation Dissertation Fellowship for Gabriela Farfan.

\section{REFERENCES}

(1) Spurgeon, J. P. G. Mar. Pollut. Bull. 1992, 24, 529-536.

(2) Dauphin, Y. Int. J. Biol. Macromol. 2001, 28, 293-304.

(3) Cuif, J. P.; Dauphin, Y. Y.; Doucet, J.; Salome, M.; Susini, J. Geochim. Cosmochim. Acta 2003, 67, 75-78.

(4) Nguyen, L. T.; Rahman, M. A.; Maki, T.; Tamenori, Y.; Yoshimura, T.; Suzuki, A.; Iwasaki, N.; Hasegawa, H. Geochim. Cosmochim. Acta 2014, 127, 1-9.
(5) Vielzeuf, D.; Garrabou, J. ; Gagnon, A.; Ricolleau, A.; Adkins, J.; Günther, D.; Hametner, K.; Devidal, J. L.; Reusser, E.; Perrin, J.; Floquet, N. Chem. Geol. 2013, 355, 13-27.

(6) Tamenori, Y.; Yoshimura, T.; Luan, N. T.; Hasegawa, H.; Suzuki, A.; Kawahata, H.; Iwasaki, N. J. Struct. Biol. 2014, 186, 214 23.

(7) Raina, J. B.; Tapiolas, D. M.; Foret, S.; Lutz, A.; Abrego, D.; Ceh, J.; Seneca, F.; Clode, P. L.; Bourne, D. G.; Willis, B. L.; Motti, C. A. Nature 2013, 502, 677-680.

(8) Present, T.; Paris, G.; Burke, A.; Fischer, W. W.; Adkins, J. F Earth Planet. Sci. Lett. 2015, 432, 187-198.

(9) Manceau, A.; Nagy, K. L. Geochim. Cosmochim. Acta 2012, 99, 206-223.

(10) Rompel, A.; Cinco, R. M.; Latimer, M. J.; McDermott, A. E.; Guiles, R. D.; Quintanilha, A.; Krauss, R. M.; Sauer, K.; Yachandra, V. K.; Klein, M. P. Proc. Natl. Acad. Sci. 1998, 95, 6122-6127.

(11) Xia, K.; Weesner, F.; Bleam, W. F.; Bloom, P. R.; Skyllberg, U. L.; Helmke, P. A. Soil Sci. Soc. Am. J. 1998, 62, 1240-1246.

(12) Cusack, M.; Dauphin, Y.; Cuif, J. P.; Salome, M.; Freer, A.; Yin, H. Chem. Geol. 2008, 253, 172-179.

(13) Dauphin, Y.; Cuif, J. P.; Salome, C.; Susini, J. Am. Mineral. 2005, 90, 1748-1758.

(14) Perrin, J.; Rivard, C.; Vielzeuf, D.; Laporte, D.; Fonquernie, C.; Ricolleau, A.; Cotte, M.; Floquet, N. Geochim. Cosmochim. Acta 2017, 197, 226-244.

(15) Templeton, A.; Knowles, E. Annu. Rev. Earth Planet. Sci. 2009, 37, 367-391.

(16) Vázquez-Rodríguez, A. I.; Hansel, C. M.; Zhang T.; Lamborg, C. H. Front. Microbiol. 2015, 6, 1-11.

(17) Mayhew, L. E.; Webb, S. M.; Templeton, A. S. Environ. Sci. Technol. 2011, 45, 4468-4472.

(18) Keiluweit, M.; Bougoure, J. J.; Nico, P. S.; Pett-Ridge, J.; Weber, P. K.; Kleber, M. Nat. Clim. Change 2015, 5, 588.

(19) Benzerara, K., Menguy, N., López-García, P., Yoon, T. H., Kazmierczak, J.; Tyliszczak, T.; Brown, G. E. Proc. Natl. Acad. Sci., 2006, 103, 9440-9445.

(20) Webb S. M. Phys. Scr. 2005, T115, 1011-1014.

(21) Webb S. M. AIP Conf. Proc. 2011, 1365, 196-199.

(22) Pingitore, N. E.; Meitzner, G.; Love, K. M. Geochim. Cosmochim. Acta 1995, 59, 2477-2483.

(23) Yoshimura, T.; Tamenori, Y.; Suzuki, A.; Nakashima, R.; Iwasaki, H. H.; Kawahara, H. Chem. Geol. 2013, 352, 170-175.

(24) Allemand, D.; Ferrier-Pagès, C.; Furla, P.; Houlbrèque, F.; Puverel, S.; Reynaud, S.; Tambutté, É.; Tambutté, S.; Zoccola, D. Comptes Rendus Palevol 2004, 3, 453-467.

(25) Allemand, D.; Tambutté, É.; Zoccola, D.; Tambutté, S. Coral Calcification, Cells to Reefs. In Coral Reefs: An Ecosystem in Transition (eds. Z. Dubinsky and N. Stambler). Springer Netherlands 2011, $119-150$.

(26) McLenon, A. L.; DiTullio, G. R. Biogeochemistry 2012, 110, $17-29$.

(27) Wegley, L.; Edwards, R.; Rodriguez-Brito, B.; Liu, H.; Rohwer, F. Environ. Microbiol. 2007, 9, 2707-2719.

(28) Takano, B.; Asano, Y.; Watanuki, K. Contrib. to Mineral. Petrol. 1980, 72, 197-203.

(29) Busenberg, E.; Plummer, N. L. Geochim. Cosmochim. Acta 1985, 49, 713-725.

(30) Fernández-Díaz, L.; Fernández-González, Á.; Prieto, M. Geochim. Cosmochim. Acta 2010, 74, 6064-6076.

(31) Takano, B. Chem. Geol. 1985, 49, 393-403.

(32) Kampschulte, A.; Strauss, H. Chem. Geol. 2004, 204, 255286.

(33) Allemand, D.; Cuif, J. P.; Watabe, N.; Oishi, M. Bull. Inst. océanogr. (Monaco) 1994, 129-140.

Authors are required to submit a graphic entry for the Table of Contents (TOC)--Insert Table of Contents artwork: 


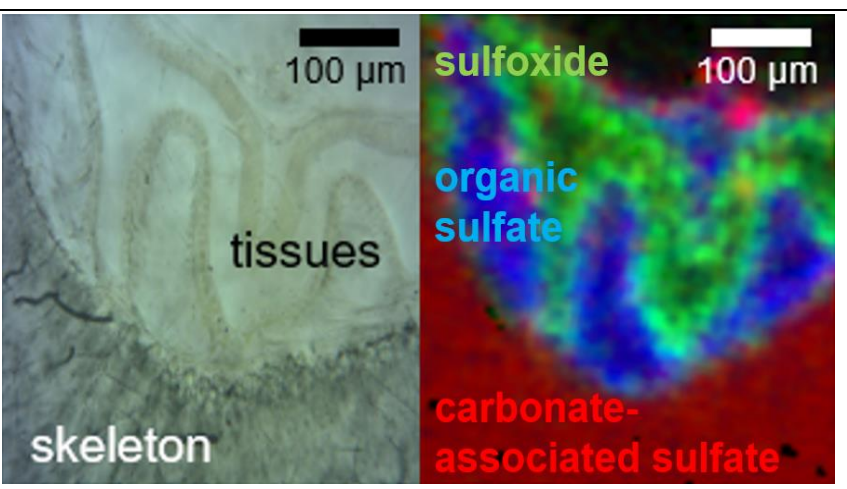

